\title{
Marshll-Olkin Extended Inverse Pareto Distribution and its Application
}

\author{
M-Gharib $^{1}$, B-I-Mohammed ${ }^{2} \&$ W-E-R-Aghel ${ }^{1}$ \\ ${ }^{1}$ Department of Mathematics, Faculty of Science, Ain-Shams University, Abbassia, Cairo, Ejypt \\ ${ }^{2}$ Department of Mathematics, Faculty of Science, Al-Azhar University, Nasr City, Cairo, Ejypt \\ Correspondence: W-E-R-Aghel, Department of Mathematics, Faculty of Science, Ain-Shams University, Abbassia, \\ Cairo, Ejypt, Ejypt. E-mail: Wesalagil@yahoo.com
}

Received: July 12, 2017 Accepted: July 28, 2017 Online Published: September 21, 2017

doi:10.5539/ijsp.v6n6p71 URL: https://doi.org/10.5539/ijsp.v6n6p71

\begin{abstract}
This paper introduces a new extension of the Inverse Pareto distribution along with in the framework of Marshal-Olkin (1997) family of distributions. This model is capable of modeling various shapes of aging and failure criteria. The statistical properties of the new model are discussed and the maximum likelihood and maximum product spacing's methods are used to estimate the parameters involved. Explicit expressions are derived for the moments and the order statistics are examined for the new proposed model. Finally, the usefulness of the new model for modeling reliability data is illustrated using two real data sets with simulation study.
\end{abstract}

Keywords: Inverse Pareto distribution, reliability analysis, maximum likelihood estimation, maximum product spacing's estimates.

\section{Introduction}

The procedure of adding one or two parameters to a family of distributions to obtain more flexible models is a well-known technique in the literature. Marshall and Olkin (1997) pioneered a simple method of adding a positive shape parameter into a family of distributions and several authors used their method to extend well-known distributions in the last few years. If $\bar{G}(x), g(x)$ and $r(x)$ denote respectively the survival function (sf), probability density function (pdf) and hazard rate function (hrf) of a parent distribution, then the survival function $\bar{F}(x)$ of the Marshall - Olkin (MO) family is defined by:

$$
\bar{F}(x ; \delta)=\frac{\delta \bar{G}(x)}{1-\bar{\delta} \bar{G}(x)},-\infty<x<\infty, \delta>0, \bar{\delta}=1-\delta .
$$

Clearly, for $\delta=1$, we obtain the baseline distribution, i.e., $\bar{F}(x)=\bar{G}(x)$.

The shape parameter $\delta$, is called "tilt parameter", since the hrf $h(x ; \alpha)$ of the transformed distribution is shifted below $(\delta \geq 1)$ or above $(0<\delta \leq 1)$ from the baseline hrf, say $r(x)$ (Marshall \& Olkin, 1997).

The pdf corresponding to (1) is given by

$$
f(x ; \delta)=\frac{\delta \mathrm{g}(x)}{\{1-\bar{\delta} \overline{\mathrm{G}}(x)\}^{2}},
$$

and its hrf reduces to

$$
h(x ; \delta)=\frac{\mathrm{r}(\mathrm{x})}{1-\bar{\delta} \bar{G}(x)} .
$$

We write $X \sim I P(\alpha, \beta)$ to denote a random variable $X$ having the two-parameter inverse Pareto (IP) distribution with cumulative distribution function (cdf) 


$$
G(x ; \alpha, \beta)=\left(\frac{x}{x+\beta}\right)^{\alpha}, x>0, \alpha>o, \beta>0,
$$

and corresponding pdf

$$
g(x ; \alpha, \beta)=\frac{\alpha \beta x^{\alpha-1}}{(\beta+x)^{\alpha+1}}, x>0, \alpha>0, \beta>0 .
$$

In this article, the IP distribution is embedded in a larger family by adding an extra shape parameter, by applying the MO technique and is called the Marshall-Olkin extended inverse Pareto (MOEIP) distribution.

The rest of this paper is outlined as follows. In section (2), we define the new distribution, derive a linear representation for its pdf, and provide some sub-models and plots of the distribution. In section (3) we introduced some statistical properties of the MOEIP distribution. In section (4) the order statistics and their moments are investigated. In section (5), we discuss the maximum likelihood estimation of the model parameters. Section (6) is devoted to give some applications of the MOEIP model.

\section{The MOEIP Distribution}

In this section, we define the survival, the pdf and the hazard rate functions of the new distribution. The cdf of the MOEIP distribution with three positive parameters $(\alpha, \beta, \delta)$ is obtained by inserting (4) into (1) a

$$
\bar{F}(x ; \alpha, \beta, \delta)=1-\frac{\delta\left[1-\left(\frac{x}{x+\beta}\right)^{\alpha}\right]}{\delta-(\delta-1)\left(\frac{x}{\beta+x}\right)^{\alpha}}, x>0, \delta>0,
$$

The MOEIP density function is given by:

$$
f(x ; \alpha, \beta, \delta)=\frac{\alpha \beta \delta x^{\alpha-1}(\beta+x)^{-\alpha-1}}{\left[\delta-(\delta-1)\left(\frac{x}{\beta+x}\right)^{\alpha}\right]^{2}},
$$

and the corresponding hrf by,

$$
h(x ; \alpha, \beta, \delta)=\frac{\tau(x, \alpha, \beta)}{\delta-(\delta-1)\left(\frac{x}{\beta+x}\right)^{\alpha}} .
$$

where $\tau(x, \alpha, \beta)$ is the hrf of the IP distribution (4).

\section{Theorem 1.}

The pdf of the MOEIP distribution given by (7) can be represented as a countable mixture of inverse Pareto distributions as:

$$
f(x ; \alpha, \beta, \delta)=\sum_{k=0}^{\infty} v_{k} g_{k+1}(x),
$$

where

$$
v_{k}=\frac{(\delta-1)^{k}}{\delta^{k+1}}, \delta>0,
$$


and

$$
g_{k}(x)=\frac{\alpha \beta x^{\alpha k-1}}{(\beta+x)^{\alpha k+1}}
$$

\section{Proof}

Appling the binomial expansion of $\left[\delta-(\delta-1)\left(\frac{x}{\beta+x}\right)^{\alpha}\right]^{-2}$, to equation (7) we can write

$$
f(x ; \alpha, \beta, \delta)=\sum_{k=0}^{\infty} v_{k} \frac{\alpha(k+1) \beta x^{\alpha(k+1)-1}}{(\beta+x)^{\alpha(k+1)+1}},
$$

where, $v_{k}$ is given by (10). Or, equivalently

$$
f(x ; \alpha, \beta, \delta)=\sum_{k=0}^{\infty} v_{k} g_{k+1}(x),
$$

where, $g_{k}(x)$ is given by (11). It is clear that (11) is the pdf of the $I P(\alpha k, \beta)$ distribution.

Also, It follows form (10) that for $\delta>1,0<v_{k}<1$ and $\sum_{k=0}^{\infty} v_{k}=1$.

One can see from (9) that the new model is a mixture of IP distributions. This completes the proof.

It follows from (9) that the different properties of the MOEIP distribution can be generally discussed through the corresponding properties of the IP distribution.

\subsection{Plots for the EMOIP Distribution}

Figures (1) and (2) below displays the plots of the MOEIP density for some selected values of the parameters $\alpha, \beta$ and $\delta$. These plots illustrate the versatility, initially decreasing and modality of this distribution, devise shapes with the changes in the parameters some show a mode, and others do not. Some show bathtub and others show monotonicity.

The plots in figures (3) and (4) below reveal that the hrf of the MOEIP distribution can have unimodal and decreasing shapes.
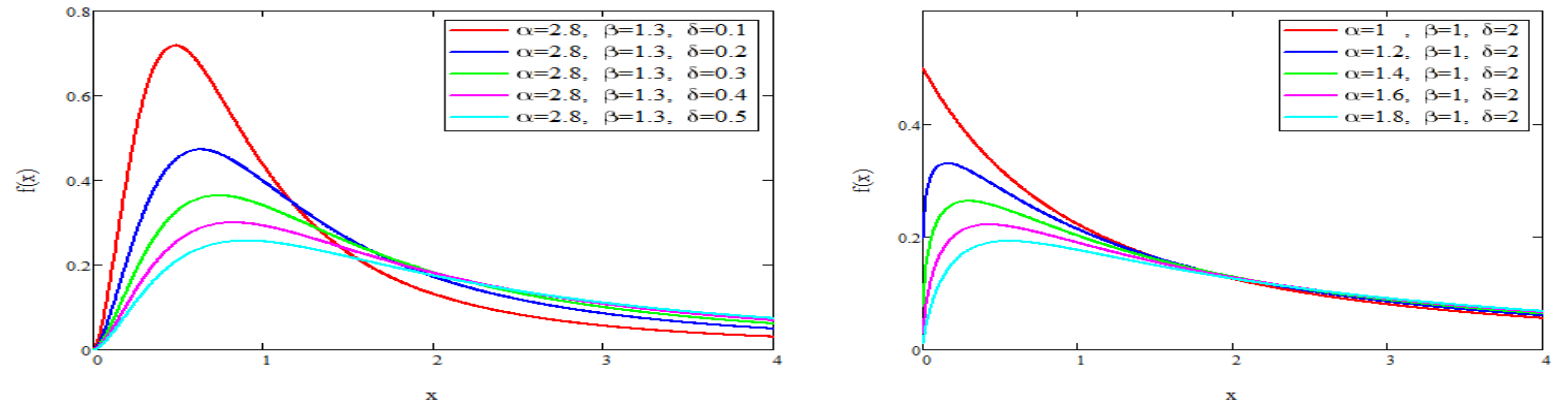

Figure 1. Plots of the MOEIP pdf for selected parameters values 

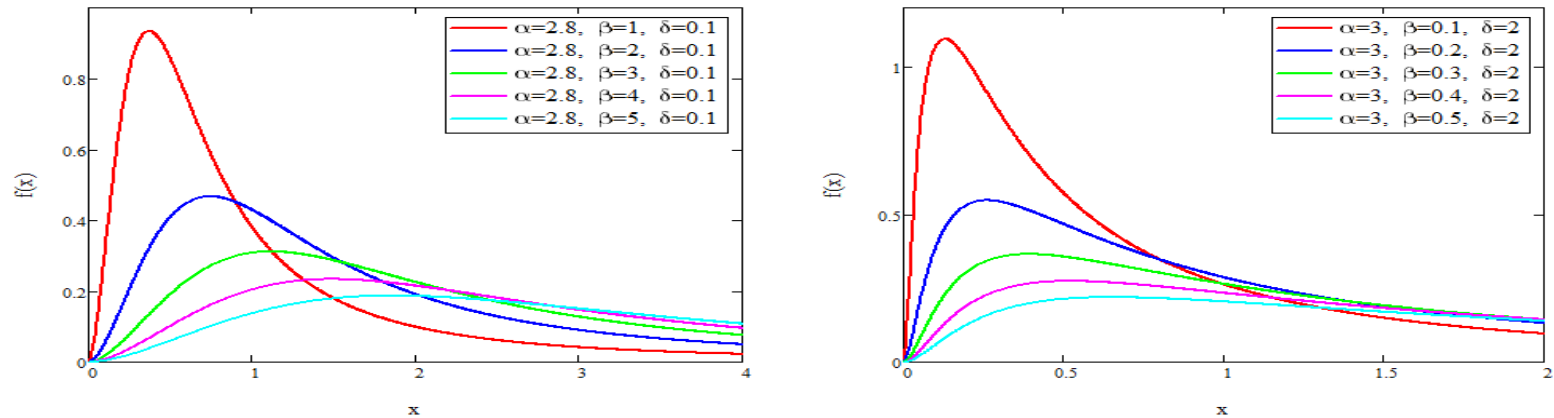

Figure 2. Plots of the MOEIP pdf for selected parameters values
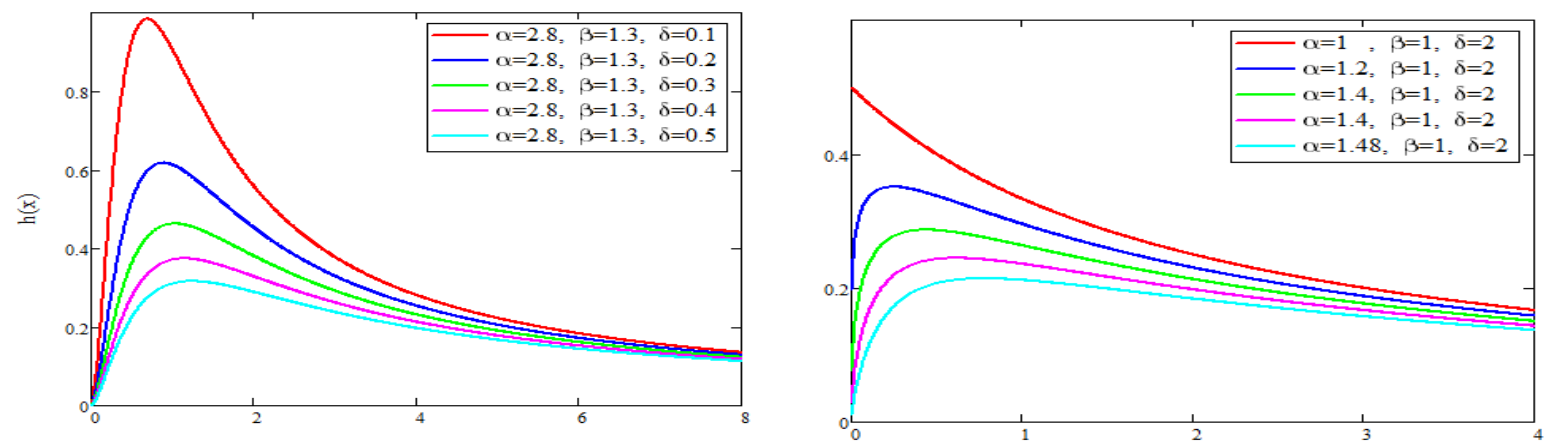

Figure 3. Plots of the MOEIP hrf for selected parameters values
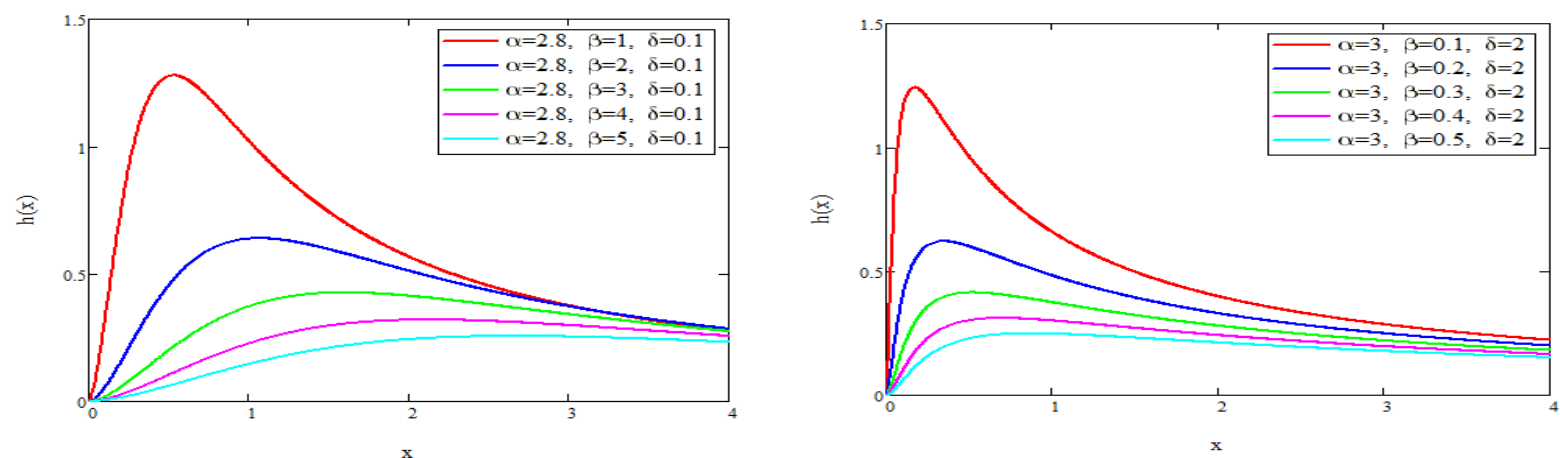

Figure 4. Plots of the MOEIP hrf for selected parameters values

The plots in figures (5) and (6) below show the behaviour of the cdf and sf of the MOEIP distribution for some selected values of the parameters. The figures show that cdf curve is initially increasing function.
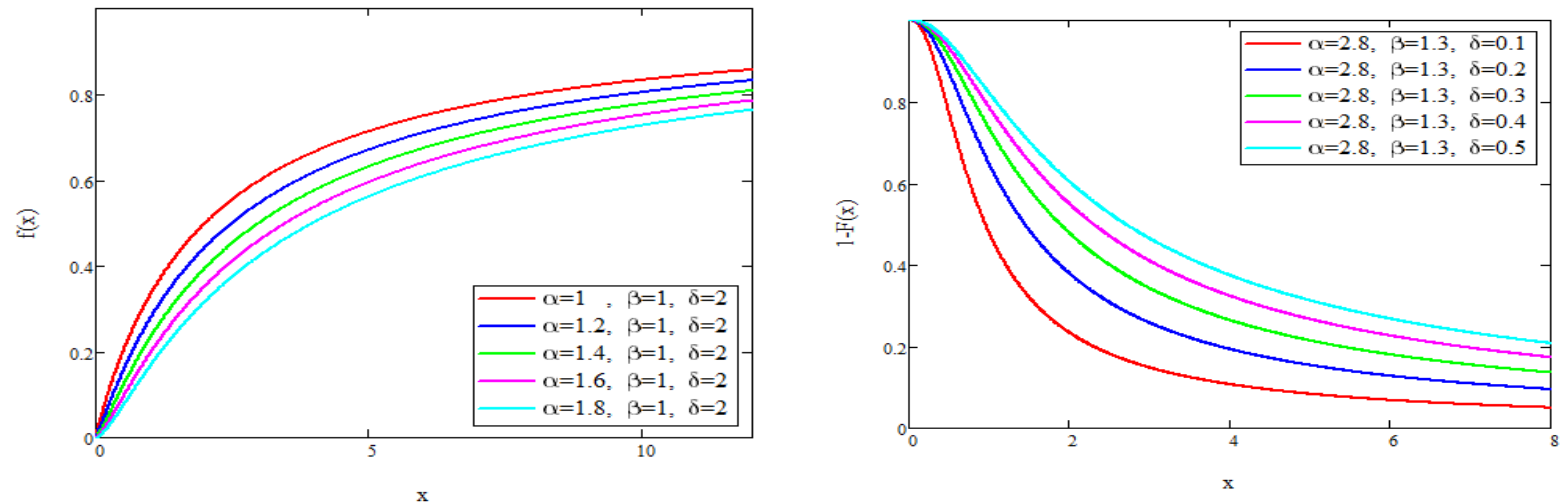

Figure 5. Plots of the MOEIP cdf and sf for selected parameters values 


\section{Statistical Properties of the MOEIP Distribution.}

\subsection{The Moments of EMOIP Distribution}

Due to its mathematical tractability the moments of different types of the MOEIP distribution can be obtained using direct calculations. The first incomplete moment has important applications related to the Bonferroni and Lorenz curves, the mean residual life and the mean waiting time. Furthermore, the amount of scatter in a population is measured to some extent by the totality of deviations from the mean and median. These three functions uniquely determine the cdf of the MOEIP distribution; depend on the first incomplete moment.

The $r$-th ordinary and incomplete moments, of the MOEIP distribution (7) are given by

$$
\mu_{r}^{\prime}=\alpha \beta^{r} B(\alpha+r, 1-r), \quad r=1,2, \ldots
$$

and

$$
\varphi_{r}(t)=\alpha \beta^{r} B\left(\frac{t}{(\beta+t)} ; \alpha+r, 1-r\right), \quad r=1,2, \ldots
$$

respectively, where $B(a, b)=\int_{0}^{1} z^{a-1}(1-z)^{b-1} d z$ ? is the beta function and

$B(y ; a, b)=\int_{0}^{y} z^{a-1}(1-z)^{b-1} d z ;$ is the incomplete beta function

Equation (12) has the following equivalent form obtained by calculating $\mu_{r}^{\prime}$ using (9).

$$
\mu_{r}^{\prime}=\sum_{k=0}^{\infty} v_{k} \alpha(k+1) \beta^{r} B(\alpha(k+1)+r, 1-r) .
$$

Now using the relation between the central and non-central moments, it is easy to obtain the $n$-th central moment $M_{n}$ of the MOEIP distribution given by (7), as

$$
M_{n}=\sum_{i=0}^{n} \sum_{k=0}^{\infty} v_{k}\left(\begin{array}{l}
n \\
i
\end{array}\right)\left(-\mu_{1}^{\prime}\right)^{n-i} \alpha(k+1) \beta^{i} B(\alpha(k+1)+i, 1-i),
$$

Equation (15) can be used to obtain the measures of skewers and kurtosis measures of the MOEIP distribution. The $r$-th negative moment of the MOEIP distribution (7) can be simply obtained as

$$
E\left(\frac{1}{x^{r}}\right)=\sum_{k=0}^{\infty} v_{k} \frac{\alpha(k+1)}{\beta^{r}} B(\alpha(k+1)-r, 1+r),
$$

the $n$-th moment of the residual life of the MOEIP distribution (7) can be expressed as

$$
m_{n}(t)=\frac{1}{\bar{F}(t)} \int_{t}^{\infty}(x-t)^{n} d F(x), n=1,2, \ldots
$$

Further, it can be expressed as

$$
m_{n}(t)=\frac{1}{\bar{F}(t)} \sum_{j=0}^{n} \sum_{k=0}^{\infty}(-1)^{n-j}\left(\begin{array}{c}
n \\
j
\end{array}\right) t^{n-j} v_{k} \alpha(k+1) \beta^{j} B\left(\frac{t}{(\beta+t)} ; \alpha(k+1)+j, 1-j\right),
$$

where $\bar{F}(x)$ is given by (6). 
$m_{1}(t)$ is the mean residual life function. It represents the expected additional life length for a unit which is alive at age $t$.

The cdf is uniquely determined by the mean reversed residual life of the MOEIP distribution, it is given by

$$
M_{n}(t)=\frac{1}{F(t)} \int_{0}^{t}(t-x)^{n} d F(x) \text {. }
$$

In a similar manner, it can be expressed as

$$
M_{n}(t)=\frac{1}{F(t)} \sum_{j=0}^{n} \sum_{k=0}^{\infty}(-1)^{j} t^{n-r}\left(\begin{array}{c}
n \\
j
\end{array}\right) v_{k} \alpha(k+1) \beta^{j} B\left(\frac{t}{(\beta+t)} ; \alpha(k+1)+j, 1-j\right) .
$$

The mean inactivity time (MIT) of MOEIP distribution is obtained by setting $n=1$ in the previous equation.

\subsection{Quantiles of the MOEIP Distribution}

The $p$-th quantile $\left(x_{p}\right)$ of the MOEIP distribution is obtained by so having equation.

$$
F\left(x_{p}\right)=p
$$

where $F(x)=1-\bar{F}(x)$. Hence solving (21) we get

$$
x_{p}=\frac{\beta}{\left[\frac{\delta p}{1+p(\delta-1)}\right]^{-\frac{1}{\alpha}}-1},
$$

the $p$-th quantile for $p \in(0,1)$.

For $p=0.5$ the median of the MOEIP distribution is given by

$$
x_{0.5}=\beta\left\{\left[\frac{0.5 \delta}{0.5+0.5 \delta}\right]^{-\frac{1}{\alpha}}-1\right\}^{-1} .
$$

Remark 1. For $\delta=1$ we have the qf of the IP distribution as

$$
x_{p}=\frac{\beta}{p^{-\frac{1}{\alpha}}-1} .
$$

\section{Distribution of Order Statistics of the MOEIP Distribution}

Let $X_{1}, \ldots, X_{n}$ be a random sample of size $\mathrm{n}$ from the MOEIP $(\alpha, \beta, \delta)$ model with sf and pdf given by (6) and (7), respectively

\section{Theorem 2.}

The pdf of the $i$-th order statistic, say $X_{i: n}, 1 \leq i \leq n$, is given by:

$$
f_{i: n}(x)=\sum_{k, l=0}^{\infty} m_{k, l} g_{k+l+1}(x)
$$

and

$$
m_{k, l}=\sum_{s=0}^{\infty} \frac{(-1)^{j+s+l} \Gamma(k+s+2)}{\delta(k+l+1) k ! B(i, n-i+1) \Gamma(s+2)}\left(\frac{\delta-1}{\delta}\right)^{k}\left(\begin{array}{c}
n-1 \\
j
\end{array}\right)\left(\begin{array}{c}
j+i-1 \\
s
\end{array}\right)\left(\begin{array}{l}
s \\
l
\end{array}\right),
$$


where $g_{k+l+1}(x)$ is the IP density function with parameters $\alpha(k+l+1)$ and $\beta$.

\section{Proof}

It is known that the pdf of the $i$-th order statistics is given:

$$
f_{i: n}(x)=\frac{f(x)}{B(i, n-i+1)} \sum_{j=0}^{n-i}(-1)^{j}\left(\begin{array}{c}
n-1 \\
j
\end{array}\right) F(x)^{j+i-1} .
$$

Using equation (7), we have

$$
F(x)^{j+i-1}=\left\{1-\frac{\delta\left[1-\left(\frac{x}{x+\beta}\right)^{\alpha}\right]}{\delta-(\delta-1)\left(\frac{x}{\beta+x}\right)^{\alpha}}\right\}^{j+i-1} .
$$

Using the generalized binomial series, we can write:

$$
F(x)^{j+i-1}=\sum_{s=0}^{\infty}(-1)^{s}\left(\begin{array}{c}
j+i-1 \\
s
\end{array}\right) \frac{\delta^{s}\left[1-\left(\frac{x}{x+\beta}\right)^{\alpha}\right]^{s}}{\left[\delta-(\delta-1)\left(\frac{x}{\beta+x}\right)^{\alpha}\right]^{s}} .
$$

Then,

$$
f(x) F(x)^{j+i-1}=\alpha \beta x^{\alpha-1}(\beta+x)^{-\alpha-1} \sum_{s=0}^{\infty}(-1)^{s}\left(\begin{array}{c}
j+i-1 \\
s
\end{array}\right) \frac{\delta^{s+1}\left[1-\left(\frac{x}{x+\beta}\right)^{\alpha}\right]^{s}}{\left[\delta-(\delta-1)\left(\frac{x}{\beta+x}\right)^{\alpha}\right]^{s+2}} .
$$

Now, using power series expansion, we have

$$
\left[\delta-(\delta-1)\left(\frac{x}{\beta+x}\right)^{\alpha}\right]^{-s-2}=\delta^{-s-2} \sum_{k=0}^{\infty} \frac{\Gamma(k+s+2)}{\Gamma(s+2) k !}\left(\frac{\delta-1}{\delta}\right)^{k}\left(\frac{x}{\beta+x}\right)^{\alpha k} .
$$

Similarly, for to the term $\left[1-\left(\frac{x}{x+\beta}\right)^{\alpha}\right]^{s}$, we have

$$
\left[1-\left(\frac{x}{x+\beta}\right)^{\alpha}\right]^{s}=\sum_{l=0}^{\infty}(-1)^{l}\left(\begin{array}{l}
s \\
l
\end{array}\right)\left(\frac{x}{x+\beta}\right)^{\alpha l} .
$$

Substituting the last two expressions in equation (25), we have

$$
E\left(X_{i: n}^{q}\right)=\sum_{k, l=0}^{\infty} m_{k, l} \alpha(k+l+1) \beta^{q} B(\alpha(k+l+1)+q, 1-q) .
$$

\section{Estimation of the Parameters of the MOEIP Distribution.}

In this section, we shall derive estimates of the parameter using two different methods. 


\subsection{Maximum Likelihood Estimates}

Consider the estimation of the unknown parameters of the MOEIP distribution (7), from complete samples by maximum likelihood. Let $x_{1}, \ldots, x_{n}$ ? be a random sample of this distribution with unknown parameter vector $v=(\alpha, \beta, \delta)^{T}$. The log-likelihood function, say $\ell=\ell(v)$ obtained from equation (7), is given by:

$$
\begin{aligned}
\ell= & n \log (\alpha)+n \log (\beta)+n \log (\delta)-(\alpha+1) \sum_{i=1}^{n} \log \left(\beta+x_{i}\right)+(\alpha-1) \sum_{i=1}^{n} \log \left(x_{i}\right) \\
& -2 \sum_{i=1}^{n} \log \left(\delta-(\delta-1)\left(\frac{x_{i}}{\beta+x_{i}}\right)^{\alpha}\right) .
\end{aligned}
$$

The components of the score vector $U(v)=\frac{\partial \ell}{\partial v}=\left(\frac{\partial \ell}{\partial \alpha}, \frac{\partial \ell}{\partial \beta}, \frac{\partial \ell}{\partial \delta}\right)^{T}$ are

$$
\begin{gathered}
\frac{\partial \ell}{\partial \alpha}=\frac{n}{\alpha}+\sum_{i=1}^{n} \log \left(x_{i}\right)-\sum_{i=1}^{n} \log \left(\beta+x_{i}\right)+2(\delta-1) \sum_{i=1}^{n} \frac{\left(\frac{x_{i}}{\beta+x_{i}}\right)^{\alpha} \log \left(\frac{x_{i}}{\beta+x_{i}}\right)}{\delta-(\delta-1)\left(\frac{x_{i}}{\beta+x_{i}}\right)^{\alpha}}=0, \\
\frac{\partial \ell}{\partial \beta}=\frac{n}{\beta}-\sum_{i=1}^{n} \frac{(\alpha+1)}{\beta+x_{i}}-2 \sum_{i=1}^{n} \frac{(\delta-1) \frac{\alpha}{x_{i}}\left(\frac{x_{i}}{\beta+x_{i}}\right)^{\alpha+1}}{\delta-(\delta-1)\left(\frac{x_{i}}{\beta+x_{i}}\right)^{\alpha}}=0,
\end{gathered}
$$

and

$$
\frac{\partial \ell}{\partial \delta}=\frac{n}{\delta}-\sum_{i=1}^{n} \frac{2-2\left(\frac{x_{i}}{\beta+x_{i}}\right)^{\alpha}}{\delta-(\delta-1)\left(\frac{x_{i}}{\beta+x_{i}}\right)^{\alpha}}=0 .
$$

We require iterative techniques such as the Newton-Raphson algorithm to solve the above equations numerically.

5.2 Maximum Product Spacing Estimates

$$
G M=\sqrt[n+1]{\prod_{i=1}^{n+1} D_{i}}
$$

where, the difference $D_{i}$ is defined as

$$
D_{i}=\int_{x_{(i-1)}}^{x_{(i)}} f(x, \alpha, \beta, \delta) d x, i=1,2, \ldots, n+1
$$

where, $\mathrm{F}(\mathrm{x}, \alpha, \beta, \delta)$ is the cdf, $F\left(x_{(0)}, \alpha, \beta, \delta\right)=0$ and $F\left(x_{(n+1)}, \alpha, \beta, \delta\right)=0$.

The MPS estimators $\hat{\alpha}_{P S}, \hat{\beta}_{P S}$ and $\hat{\delta}_{P S}$ of $\alpha, \beta$ and $\delta$ are obtained by maximizing the geometric mean (GM) of the differences. Substituting pdf of MOEIP and taking logarithm of the above expression, we will have 


$$
\begin{aligned}
& \frac{\partial \log G M}{\partial \alpha}=\frac{1}{n+1} \sum_{i=1}^{n+1}\left[\frac{F_{\alpha}^{\prime}\left(x_{(i)}, \alpha, \beta, \delta\right)-F_{\alpha}^{\prime}\left(x_{(i-1)}, \alpha, \beta, \delta\right)}{F\left(x_{(i)}, \alpha, \beta, \delta\right)-F\left(x_{(i-1)}, \alpha, \beta, \delta\right)}\right]=0, \\
& \frac{\partial \log G M}{\partial \beta}=\frac{1}{n+1} \sum_{i=1}^{n+1}\left[\frac{F_{\beta}^{\prime}\left(x_{(i)}, \alpha, \beta, \delta\right)-F_{\beta}^{\prime}\left(x_{(i-1)}, \alpha, \beta, \delta\right)}{F\left(x_{(i)}, \alpha, \beta, \delta\right)-F\left(x_{(i-1)}, \alpha, \beta, \delta\right)}\right]=0,
\end{aligned}
$$

and

$$
\frac{\partial \log G M}{\partial \delta}=\frac{1}{n+1} \sum_{i=1}^{n+1}\left[\frac{F_{\delta}^{\prime}\left(x_{(i)}, \alpha, \beta, \delta\right)-F_{\delta}^{\prime}\left(x_{(i-1)}, \alpha, \beta, \delta\right)}{F\left(x_{(i)}, \alpha, \beta, \delta\right)-F\left(x_{(i-1)}, \alpha, \beta, \delta\right)}\right]=0 .
$$

where,

$$
F_{\alpha}^{\prime}\left(x_{(i)}, \alpha, \beta, \delta\right)=-\frac{\left[1-\left(\frac{x}{\beta+x}\right)^{\alpha}\right] \cdot\left(\frac{x}{\beta+x}\right)^{\alpha}}{\left[\delta-(\delta-1)\left(\frac{x}{\beta+x}\right)^{\alpha}\right]^{2}}, F_{\beta}^{\prime}\left(x_{(i)}, \alpha, \beta, \delta\right)=-\frac{\alpha \delta \cdot\left(\frac{x}{\beta+x}\right)^{\alpha} \cdot\left(\frac{1}{\beta+x}\right)}{\left[\delta-(\delta-1)\left(\frac{x}{\beta+x}\right)^{\alpha}\right]^{2}},
$$

and

$$
F_{\delta}^{\prime}\left(x_{(i)}, \alpha, \beta, \delta\right)=\frac{\delta \cdot\left(\frac{x}{\beta+x}\right)^{\alpha} \cdot \ln \left(\frac{x}{\beta+x}\right)}{\left[\delta-(\delta-1)\left(\frac{x}{\beta+x}\right)^{\alpha}\right]^{2}} .
$$

\section{Applications of the MOEIP Distribution}

In this section, we use simulated data and real data (censored and uncensored) sets to compare the fits of the MOEIP distribution model and illustrate its usefulness.

\subsection{Simulation Study}

To assess the behavior of the maximum likelihood estimators of the parameters $\alpha, \beta$ and $\delta$ under the finite samples, we construct a Monte Carlo simulation. All results were obtained from 4000 Monte Carlo replications and the simulations were carried out using the statistical software package R.

In each replication a random sample of size $\mathrm{n}$ is drawn from the MOEIP distribution (7), the L-BFGS-B method (is a standard method for solving large instances of when the function is a smooth function, typically twice differentiable stands for bounds Broyden, Fletcher, Goldfarb and Shanno, the originators of the method The L-BFGS-B algorithm is implemented in a $\mathbf{R}$ software package) has been used to maximize the log-likelihood function. The true parameter values used in the data generating processes are $\alpha=2.8, \beta=1.3$ and $\delta=0.1$. Table 1 below, presents the mean maximum likelihood estimates of the parameters, the bias and the root mean squared errors (RMSE) for different samples of sizes $n=50, n=80$ and $n=100$.

Table 1. Mean estimates, bias and root mean squared errors of $\alpha, \beta$ and $\delta$

\begin{tabular}{c|c|c|c|c}
\hline$N$ & Parameter & Mean estimate & Bias & RMSE \\
\hline \multirow{2}{*}{$n=50$} & $\alpha$ & 8.82774 & -6.02774 & 15.04812 \\
\cline { 2 - 5 } & $\beta$ & 2.162646 & -0.862646 & 3.32376 \\
\hline
\end{tabular}




\begin{tabular}{c|c|c|c|c}
\hline & $\delta$ & 0.2102702 & -0.1102702 & 0.27109 \\
\hline \multirow{3}{*}{$n=80$} & $\alpha$ & 5.689175 & -2.889175 & 8.93957 \\
\cline { 2 - 5 } & $\beta$ & 1.972225 & -0.672225 & 2.54402 \\
\cline { 2 - 5 } & $\delta$ & 0.1702503 & -0.0702503 & 0.19429 \\
\hline \multirow{3}{*}{$n=100$} & $\alpha$ & 5.104291 & -2.304291 & 7.49537 \\
\cline { 2 - 5 } & $\beta$ & 1.912897 & -0.612897 & 2.39404 \\
\hline
\end{tabular}

We notice from Table 1. the bias and root mean squared error of the maximum likelihood estimators of $\alpha, \beta$ and $\delta$ decay toward zero as the sample size increases.

\subsection{Remission Times of a Bladder Cancer Patients - Uncensored}

The following data represents an uncensored data set corresponding to remission times (in months) of a random sample of 128 bladder cancer patients reported in Lee and Wang (2003). The data are:

$0.08,2.09,3.48,4.87,6.94,8.66,13.11,23.63,0.20,2.23,3.52,4.98,6.97,9.02,13.29,0.40,2.26,3.57,5.06,7.09,9.22$, $13.80,25.74,0.50,2.46,3.64,5.09,7.26,9.47,14.24,25.82,0.51,2.54,3.70,5.17,7.28,9.74,14.76,26.31,0.81,2.62$, $3.82,5.32,7.32,10.06,14.77,32.15,2.64,3.88,5.32,7.39,10.34,14.83,34.26,0.90,2.69,4.18,5.34,7.59,10.66,15.96$, $36.66,1.05,2.69,4.23,5.41,7.62,10.75,16.62,43.01,1.19,2.75,4.26,5.41,7.63,17.12,46.12,1.26,2.83,4.33,7.66$, $11.25,17.14,79.05,1.35,2.87,5.62,7.87,11.64,17.36,1.40,3.02,4.34,5.71,7.93,11.79,18.10,1.46,4.40,5.85,8.26$, $11.98,19.13,1.76,3.25,4.50,6.25,8.37,12.02,2.02,3.31,4.51,6.54,8.53,12.03,20.28,2.02,3.36,6.76,12.07,21.73$, $2.07,3.36,6.93,8.65,12.63,22.69$ and 5.49 .

Table 2 below presents basic descriptive statistics for the remission time data set.

Table 2. Descriptive statistic of the remission times of a bladder cancer patients

\begin{tabular}{c|c|c|c|c|c}
\hline Min. & 1st & Me & M & 3rd & Max \\
\hline 0.080 & 3.348 & 6.395 & 9.366 & 11.840 & 79.050 \\
\hline
\end{tabular}

The TTT plot for remission times of a bladder cancer patient's data is displayed in Figure 6 which provides evidence that a bathtub hazard rate is adequate.

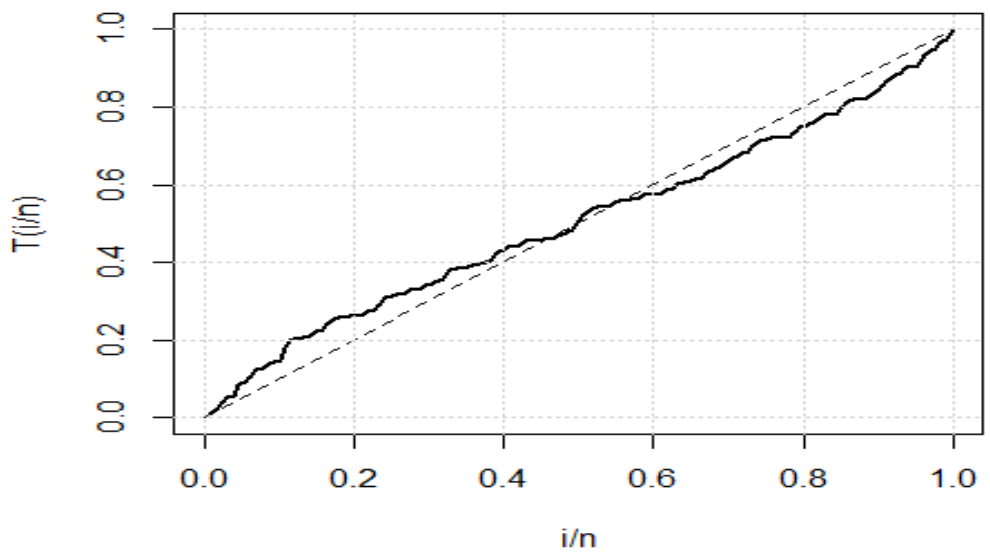

Figure 6. The TTT plot of the remission times of a bladder cancer patients

We compare the fitting of the MOEIP distribution with 8 non-nested models, this models are: the Weibull-Power series (W-Ps) distribution (Tahir et al., 2014), the new modified Weibull (NMW) distribution (Almalki \& Yuan, 2013), the additive Weibull (AW) distribution (Xie \& Lai, 1996), the Transmuted Weibull (T-W) distribution (Aryal \& Tsokos, 2011), the Kumaraswamy Pareto (Kw-P) distribution (Bourguignon et al., 2013), the exponentiated generalized Frechet (E-GF) distribution (Cordeiro et al., 2013), the Exponential-Weibull (E-W) distribution (Cordeiro et al., 2014) and the Complementary Burr III Poisson (C-BIII-P) distribution (Hassan et al., 2015).

\subsection{Model Selection}

In each case, the parameters are estimated by the maximum likelihood method and also model selection is carried out 
using log-likelihood function evaluated at the $\operatorname{MLEs}(\hat{\ell})$, Akaike information criterion (AIC), consistent Akaike information criterion (CAIC), Hannan-Quinn information criterion (HQIC), Bayesian information criterion (BIC), Anderson-Darling $\left(A^{*}\right)$, Cram'er-von Mises $\left(W^{*}\right)$ and Kolmogorov Smirnov (K-S) statistics to compare the fitted models. The statistics $A^{*}$ and $W^{*}$ are well-defined by Chen and Balakrishnan (1995). The calculation carried out using the $\mathrm{R}$ code (AdequacyModel). In general, the smaller the values of these statistics, the better the fit to the data.

These statistics are, respectively, given by:

$$
\begin{gathered}
A I C=-2 \hat{\ell}+2 k, \\
W^{*}=\left(\frac{0.5}{n}+1\right)\left[\sum_{i=1}^{n}\left(z_{i}-\frac{2 i-1}{n}\right)^{2}+\frac{1}{12 n}\right], \quad K-S=\operatorname{Max}\left[\frac{i}{n}-z_{i}, z_{i}-\frac{i-1}{n}\right],
\end{gathered}
$$

where $\hat{\ell}$ denotes the maximized log-likelihood function, $k$ is the number of estimated parameters, $n$ is the sample size, $z_{i}=c d f\left(y_{(i)}\right)$ and the $y_{(i)}$ 's are the ordered observations.

The estimates of the parameterrs and the standard error values of these estimates are listed in Table 3 while Table 4 , gives the rest of the statistics as $-\ell, \mathrm{AIC}, W^{*}$ and $\mathrm{K}-\mathrm{S}$ values.

\begin{tabular}{|c|c|c|c|c|c|}
\hline Model & \multicolumn{5}{|c|}{ Estimates } \\
\hline MOEIP & $\begin{array}{c}\alpha=1.7694 \\
(0.1556)\end{array}$ & $\begin{array}{c}\beta=107.7835 \\
(45.9225)\end{array}$ & $\begin{array}{c}\delta=0.0056 \\
(0.0027)\end{array}$ & & \\
\hline W-Ps & $\begin{array}{c}\alpha=9.7237 \\
(5.2316)\end{array}$ & $\begin{array}{c}b=0.08284 \\
(2.3151)\end{array}$ & $\begin{array}{c}\beta=12.5913 \\
(8.9562)\end{array}$ & $\begin{array}{c}\alpha=84.7241 \\
(0.2011)\end{array}$ & \\
\hline N-MW & $\begin{array}{c}\alpha=0.0763 \\
(0.01674)\end{array}$ & $\begin{array}{c}\beta=0.0176 \\
(0.0132)\end{array}$ & $\begin{array}{c}\theta=1.0476 \\
(0.0882)\end{array}$ & $\begin{array}{c}\gamma=1.0479 \\
(0.2544)\end{array}$ & $\begin{array}{l}\lambda=1 \mathrm{e}-10 \\
(0.00023)\end{array}$ \\
\hline AW & $\begin{array}{c}\alpha=1 \mathrm{e}-10 \\
(1.1144 \mathrm{e}-10)\end{array}$ & $\begin{array}{c}\theta=0.0939 \\
(0.0191)\end{array}$ & $\begin{array}{c}\beta=0.75699 \\
(\mathrm{NaN})\end{array}$ & $\begin{array}{c}\gamma=1.0478 \\
(0.0675)\end{array}$ & \\
\hline TW & $\begin{array}{c}\beta=1.0478 \\
(0.0676)\end{array}$ & $\begin{array}{c}\alpha=9.5607 \\
(0.8529)\end{array}$ & $\begin{array}{c}\theta=1 \mathrm{e}-10 \\
(0.0620)\end{array}$ & & \\
\hline $\mathrm{Kw}-\mathrm{P}$ & $\begin{array}{c}\alpha=27.2081 \\
(8.1380)\end{array}$ & $\begin{array}{c}b=15.5401 \\
(7.5091)\end{array}$ & $\begin{array}{c}\alpha=0.31896 \\
(0.0532)\end{array}$ & $\begin{array}{c}\beta=0.0055 \\
(0.0016)\end{array}$ & \\
\hline EGF & $\begin{array}{c}\alpha=81.1087 \\
(33.5614)\end{array}$ & $\begin{array}{c}\beta=0.6250 \\
(0.3010)\end{array}$ & $\begin{array}{c}\lambda=0.2816 \\
(0.0343)\end{array}$ & $\begin{array}{c}\theta=2323.1237 \\
(\mathrm{NaN})\end{array}$ & \\
\hline E-W & $\begin{array}{c}\beta=1 \mathrm{e}-10 \\
(\mathrm{NaN})\end{array}$ & $\begin{array}{c}\lambda=0.1068 \\
(0.0094)\end{array}$ & $\begin{array}{c}\alpha=0.2055 \\
(\mathrm{NaN})\end{array}$ & & \\
\hline C-BIII-P & $\begin{array}{c}\alpha=1.2853 \\
(0.0783)\end{array}$ & $\begin{array}{c}\beta=1.5895 \\
(0.4502)\end{array}$ & $\begin{array}{c}\lambda=4.5730 \\
(1.1499)\end{array}$ & & \\
\hline
\end{tabular}

Table 3. MLEs (standard errors in parentheses) to the remission times of a bladder cancer patients

Table 3 shows that MOEIP distribution fitted the data better than the other competitive models.

Table 4. The measures $-\hat{\ell}, \mathrm{AIC}, W^{*}$ and K-S to the remission times of a bladder cancer patients

\begin{tabular}{c||c|c|c|c}
\hline Model & $-\ell$ & AIC & $W^{*}$ & K-S \\
\hline \hline MOEIP & 412.7917 & 831.5835 & 0.06424052 & 0.039836, \\
& & & & p-value $=0.9872$ \\
\hline W-Ps & 413.9184 & 835.8368 & 0.133707 & 0.070259, \\
& & & & p-value $=0.5525$ \\
\hline
\end{tabular}




\begin{tabular}{c||c|c|c|c}
\hline N-MW & 414.0869 & 838.1738 & 0.1313599 & $\begin{array}{c}0.069991, \\
\text { p-value }=0.5575\end{array}$ \\
\hline AW & 414.0869 & 836.1738 & 0.1313628 & $\begin{array}{c}0.069993, \\
\text {-value }=0.5574\end{array}$ \\
\hline TW & 414.0869 & 834.1738 & 0.1313741 & $\begin{array}{c}0.070017, \\
\text { p-value }=0.557\end{array}$ \\
\hline Kw-P & 416.8697 & 841.7394 & 0.1496548 & $\begin{array}{c}0.063182, \\
\text { p-value }=0.6864\end{array}$ \\
\hline EGF & 411.9553 & 831.9106 & 0.06390148 & $\begin{array}{c}0.053263, \\
\text {-value }=0.8608\end{array}$ \\
\hline E-W & 414.3419 & 834.6838 & 0.1192921 & $\begin{array}{c}0.084637, \\
\text { - } \text {-value }=0.3183\end{array}$ \\
\hline C-BIII-P & 416.4641 & 838.9282 & 0.1356225 & $\begin{array}{c}0.49197, \\
\text { p-value }<2.2 \mathrm{e}-16\end{array}$ \\
\hline
\end{tabular}

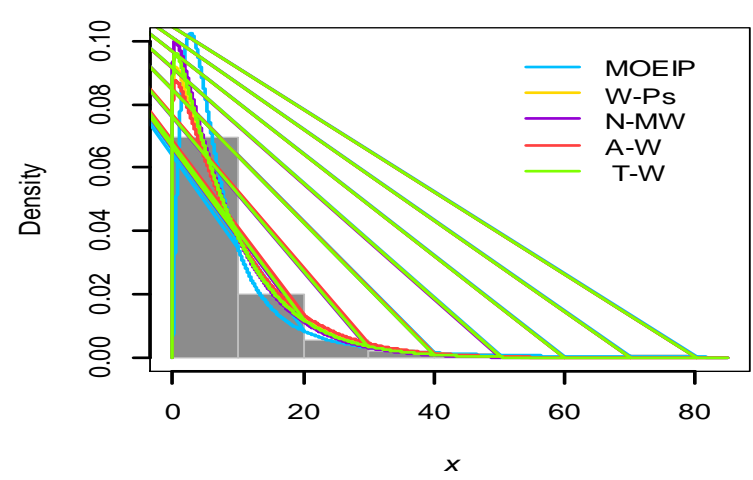

(a)

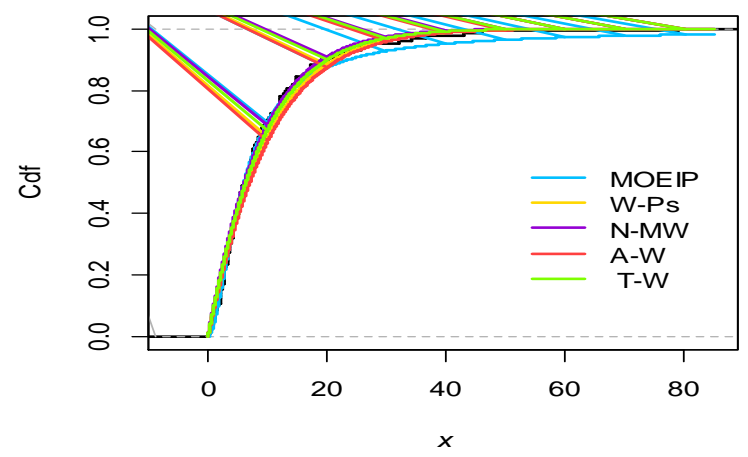

(b)

Figure 7. (a) estimated densities of the MOEIP, W-PS, N-MW, A-W and T-W distributions for the data.

(b) estimated cdf function from the fitted MOEIP, W-PS, N-MW, A-W and T-W distributions and the empirical cdf for the data.

In order to assess if the model is appropriate, we plot in Figure 7. (a) and (b) the histogram of the data and the MOEIP, W-PS, N-MW, A-W and T-W distributions and the empirical and their estimated cdf functions, respectively. These plots indicate that the MOEIP distribution provides a better fit to these data than all its sub-models.

In addition, Figure 8 (a), (b), (c), (d) and (e) present the probability-probability (PP) plot for the MOEIP, W-PS, N-MW, A-W and T-W distributions which specified used to determine how well a specific distribution fits to the observed data. This plot will be approximately linear if the specified theoretical distribution is the correct model. 


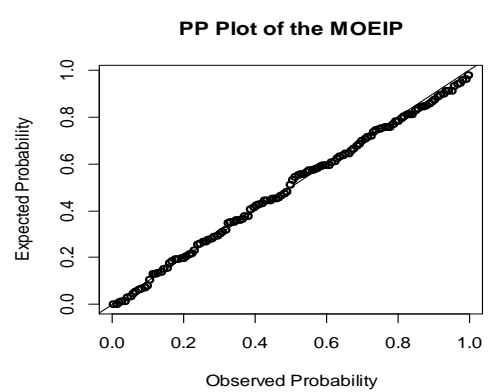

(a)

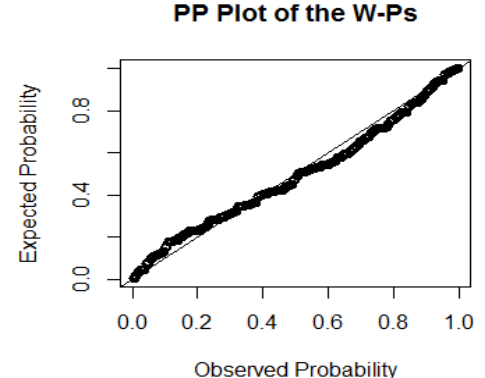

(b)

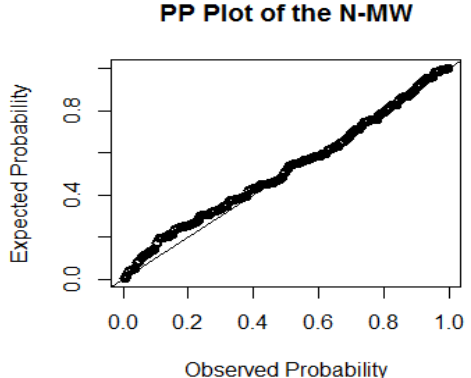

(c)
PP Plot of the A-W

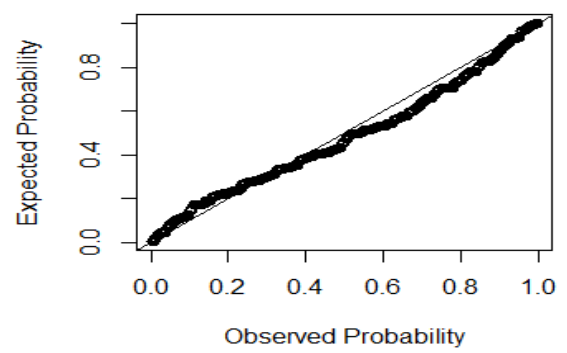

(d)

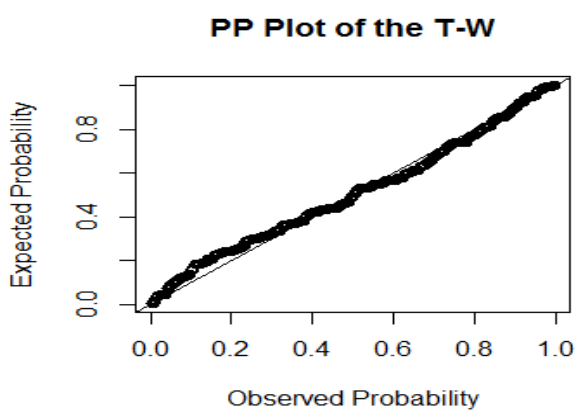

(e)

Figure 8. (a), (b), (c), (d) and (e) are the PP plot for the MOEIP, W-PS, N-MW, A-W and T-W distributions respectively

\section{Conclusion Remarks}

A new three-parameter generalization of the inverse Pareto model, called the Marshal-Olkin extended inverse Pareto, (MOEIP) distribution is defined and some of its mathematical properties studied. They include central and non-central moments, quantiles, reliability, the $\mathrm{n}$-th moment of the residual life and the $\mathrm{n}$-th moment of reversed residual life. We use two methods of estimations as the maximum likelihood and the maximum producting spacing methods to derive the equations used to maximize the likelihood function. Using the maximum likelihood method of estimation, the usefulness of the new model for modeling reliability data is illustrated using real data set with simulation study.

\section{References}

Alban, I. B, Meerschaert, M. M., \& Panorama, A. K. (2006). Parameter Estimation for the truncated Pareto distribution. Journal of the American Statistical Association, 101, 270-277. https://doi.org/10.11981016214505000000411.

Almalki, S. J., \& Yuan, J. (2013) .The new modified weibull distribution. Reliability Engineerling and System Safety, 111, 164-170. https://doi.org/10.1016/j.ress.2012.10.018

Akinsete, A., Foamy, F., \& Lee, C. (2008). The beta-Pareto distribution. Statistics, 42, 547-563. https://doi.org/10.1080/02331880801983876

Arnold, B. C. (1983). Pareto Distribution. International Co-operative Publishing House, MD, USA.

Aryal, G. R., \& Tsokos, C. P. (2011). The Transmuted Weibull distribution. Journal of Statistical Computation and Simulation, 81(10), 11-32.

Bourguignon, M, Rodrigo, B. S., Luz, M. Z., \& Gauss, M. C. (2013). The Kumaraswamy pareto distribution. Journal of Statistical Theory and Applieations, 12(2), 129-144. https://doi.org/10.1016li.csda.2008.08.023.

Carrasco. J. M. F, Ortega, E. M. M., \& Cordelier, G. M. (2008). A generalized modified Weibull distribution for lifetime modeling. Computational Statistics and Data Analysis, 53(2), 450-462. https://doi.org/10.1016/j.csda.2008.08.023

Cordeiro, G. M. E., Ortega, E. M. M., \& Dacunha, D. C. (2013). The exponentiated generalized frechet distribution. Journal of Data Science, 11, 1-27.

Cordeiro, G. M., Ortega, E. M. M., \& Lemonte, A. J. (2014). The Exponential-Weibull distribution. Journal of Statistical Computation and Simulation, 84(12), 2592-2606. https://doi.org/10.1080\0094965 s.2013.797982. 
Gail, M. H., \& Pfeiffer, R. M. (2005). On criteria for evaluating models of absolute risk, Biostatistics, 6(2),227-239, https://doi.org/10.1093lbiostatistics tkxi005.

Gutenberg, B., \& Richter, C. F. (1944). Frequency of earthquakes in California., Bulletin of the Seismological Society of America, 34, 185-188.

Hassan, A. S., Ahamed, A. M., Mokhtar, A. H. (2015). The Complementary Burr 111 Poisson distribution. Australian Journal of Basic and Applied Seiences, 9(11), 219-228.

Hogg, R. V., Hckean, J. W., \& Craig, A. T. (2005). Introduction to Mathematical Statistics. 6th ed. Pearson Prentice-Hall, New Jersey.

Jayadev, A. (2008). A power law tail in India's wealth distribution: Evidence from survey data, Physic, A: Statistical Mechanics and its Applications, 387(1), 270-276. https://doi.org/10.1016łj.physa.2007.08.049.

Jones, M. C. (2004). Families of distributions arising from distributions of orders statistics. TEST, 13, 1-43. https://doi.org/10.1007/BF02602999

Klaas, O. S, Biham, O., Levy, M., Malachi, O., \& Solomon, S. (2006). The Forbes 400 and the Pareto wealth distribution. Economics Letters, 90, 290-295. https://doi.org/10.1016/j.econlet.2005.08.020

Mackay, E. B. L., \& Challenor, P. G. (2011). “A comparison of estimators for the generalized Pareto distribution”, Ocean Engineering, 38, 1338-1346. https://doi.org/10.1016/j.oceaneng.2011.06.005

Marshall, A. M., \& Olik, I. A. (1997). New method for adding a parameter to a family of distributions with application to the exponential and weibull famines. Biometrika, 84, 641-652. https://doi.org/10.1093/biomet/84.3.641

Lucent, A. (2006). Fitting the generalized Pareto distribution to data using maximum goodness-of-fit estimators. Computational Statistics \& Data Analysis, 51, 904-917. https://doi.org/10.1016/j.csda.2005.09.011

Malamud, B. D., \& Truscott, D. L. (2006). The applicability of power-law frequency statistics to floods. Journal of Hydrology, 322, 168-180. https://doi.org/10.1016/j.jhydrol.2005.02.032

Pang, L., \& Welsh, A. H. (2001). "Robust estimation of the generalized Pareto distribution", EXTREMES, 4 (1) 53-65.

Pollard. D. (1980). The minimum distance method of testing, Metrika,27,43-70.

Reed, W. J. (2001). The Pareto, Zapf and other power laws. Economics Letter, 74, 15-19. https://doi.org/10.1016/S0165-1765(01)00524-9

Reed, W. J. (2003).The Pareto law of incomes -an explanation and an extension. Physica, Elsevier, 319, 469-486

Sinha, S. (2006). Evidence for power-law tail of the wealth distribution in India, Physica, Elsevier 359,555-562.

Tahir, M. H., Morad, A., Mansoor, M., Gauss, M. C., \& Zubair, M. (2014). The Weibull-Power series distribution with applications. Hacettepe Journal Mathematics and statistics, 45(1), 245-265 .

Ulussever, T., Guranyumusak, I., \& Kara, M. (2011). "The Day of the week effect in the Saudi stock exchange: a non linear Graph analysis". Journal of Economic and Social Studies, 1(1), 10-23.

Xie, M., \& Lai, C. D. (1996). Reliability analysis using an additive Weibull model with bathtub-shaped failure rete function. Reliability Engineering and System Safety, 52, 87-93. https://doi.org/10.1016/0951-8320(95)00149-2

Zanette, D. H., \& Manrubia, S. C. (2001). Vertical transmission of culture and the distribution of family names. Physic, 295, 1-8. https://doi.org/10.1016/S0378-4371(01)00046-2

Zaninetti, L., \& Ferraro, M. (2008). On the truncated Pareto distribution with application. Central European Journal of Physics, 6(1), 1-6. https://doi.org/10.2478/s11534-008-0008-2

\section{Copyrights}

Copyright for this article is retained by the author(s), with first publication rights granted to the journal.

This is an open-access article distributed under the terms and conditions of the Creative Commons Attribution license (http://creativecommons.org/licenses/by/4.0/). 